\title{
The Influence of Tail Fitting of Truck Effect Samples on Maximum Truck Effect in Reference Period
}

\author{
Junli Liu' ${ }^{1}$, Jinhao Zhang ${ }^{2}$, Lei Wang ${ }^{2}$, Wencheng Yu ${ }^{1,2}$ \\ 1.GuangXi Key Laboratory of Geomechanics and Geotechnical Engineering, Guilin University of \\ technology, Guilin Guangxi 541004, China; \\ 2.School of Civil Engineering and Architecture, Guilin University of Technology, Guilin Guangxi \\ 541004, China;
}

Keywords: bridge engineering; vehicle load model; tail fitting; weigh_in_motion system.

\begin{abstract}
In order to study the influence of the tail of the fitted sample distribution curve of vehicle load effect on the maximum value distribution in the reference period of vehicle load effect, multi-model normal distribution curve, straight line, parabola, exponential curve and POT model based on Pareto distribution are used respectively to fit the distribution curve of vehicle load effect sample, and the maximum distribution of different reference periods is calculated by using extreme value theory. The results show that the multi-model normal distribution model cannot accurately simulate the tail of the sample frequency curve of vehicle load effect, which can cause greater error in the extrapolation of the extreme value of the vehicle load effect, because the key is to fit exactly the tail of the sample probability distribution curve, and the tail trend of sample probability distribution curve will determine the extrapolation result of the extreme value of vehicle load effect.
\end{abstract}

\section{Introduction}

Vehicle load is the main variable load borne by the highway bridge, and it has important impact on the safety and durability of the bridge. In recent years, the development of WIM technology can obtain traffic data without affecting the normal driving condition of the vehicle, including vehicle weight, axle load and wheel base, etc., and it provides a solid foundation for the research of vehicle load model [1]. But at the present, we only obtain the short-term vehicle sample data, the vehicle load has high randomness and strong time-varying property, degeneration, makes the load response and its distribution also has a high uncertainty, and the reliability analysis and design of highway bridge need to design maximum distribution of reference period [2]. This need to be based on the short-term vehicle load effect data to deduce the long-period maximum probability distribution, the acquisition of bottom distribution of vehicle load effect samples is key to extreme extrapolation.

Currently vehicle load model of China's highway bridge [3] use Weibull distribution and logarithmic normal distribution as the bottom cumulative distribution function, extreme distribution of vehicle load is designed based on the classic extreme value theory extrapolation in reference period. Mei Gang, Qin Quan and Lin Daojin [4] use the multimodal normal distribution to describe the sample distribution, which can better describe the overall distribution of the sample data, but it cannot accurately describe the distribution of the tail. Gong Jinxin and so on [5-6] use piecewise function to fit the distribution of vehicle load, and the tail distribution of a normal distribution IS used to fit the tail of the sample.

Han Dajian [7] found that the extreme value distribution of vehicle load effect was only related to the tail distribution of the sample, and it was not related to the part near mean value. The distribution of vehicle load effect has typical fat-tailed features [8-9], and it compares with normal distribution, the tail of density curve is thicker, the curve fall more slowly, the extreme is more likely to occur, the common distribution functions such as normal distribution, the Gamma distribution and Weibull distribution can better describe the overall distribution of the sample, but the tail data is difficult to fit accurately. Li Zhihuai [9], Yuan Weizhang and so on are based on the model of the peaks over threshold method, and the Pareto distribution is used to describe the tail distribution of the sample that exceeds the threshold value. 
In order to study the effect of tail distribution fitting of vehicle load effect sample on designing maximum in reference period, this paper uses the $111 \mathrm{~d}$ WIM traffic data of a bridge to analyze the truck effect, and applies different methods to fit the tail distribution of samples.

\section{Multimodal Normal Distribution Model}

Many studies have shown that [4-5]: vehicle weights generally present the multimodal distribution characteristics, this is mainly because the traffic consists of light vehicle, midsize vehicles, heavy vehicles and so on, and there are empty load, light load, overload and other load forms, multimodal normal distribution fitting can be used. The WIM data of 111 days of a highway were adopted in this paper, and a total of 36,821 trucks with four axes or more were selected for studying. The mid-span bending moment of $6 \mathrm{~m}$ and $16 \mathrm{~m}$ standard span bridges is calculated by using the bar finite element method. The histogram of the mid-span bending moment is shown in Figure.1. As can be seen from Figure.1, the histogram of $16 \mathrm{~mm}$ mid-span vehicle load effect presents typical bimodal distribution, while the characteristics of histogram of the $6 \mathrm{~m}$ span vehicle load effect are not obvious. This is because the vehicle load effect of small span bridge is not only related to vehicle weight, and it is also affected by the factors such as axle load, wheelbase and vehicle length, the smaller the span, and it is more affected by factors such as axle load, wheelbase and vehicle length.

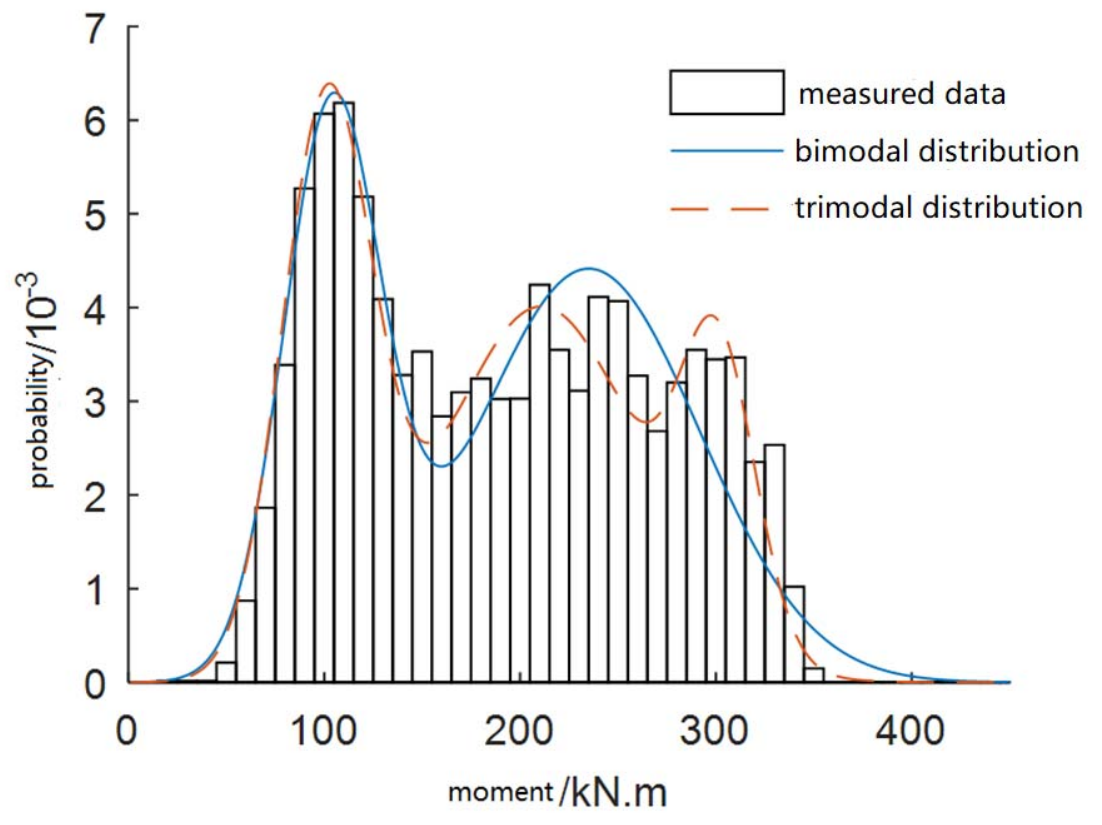

a) $6 \mathrm{~m}$ span 


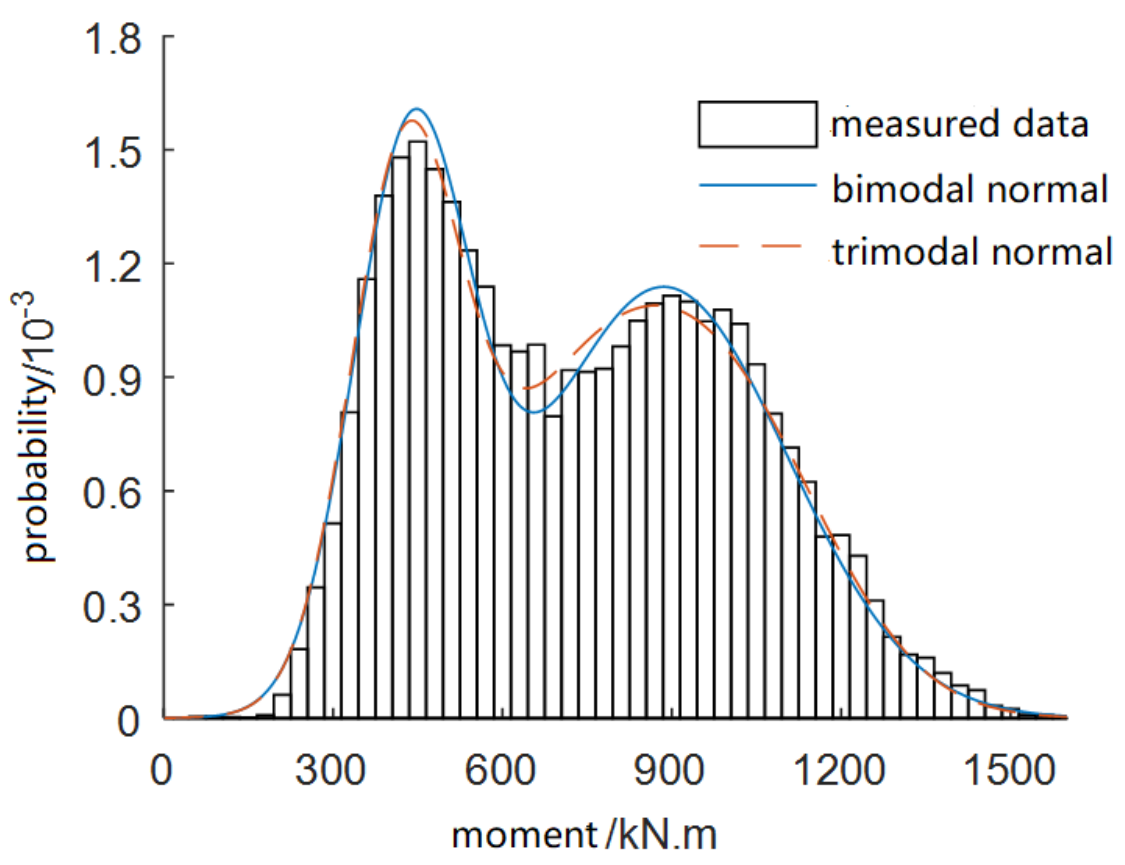

b) $16 \mathrm{~m}$ span

Fig.1 Histogram and probability density curve for truck effect

First, the multimodal normal distribution function is used to fit the vehicle load effect samples with $6 \mathrm{~m}$ and $16 \mathrm{~m}$ spans, the density function is as follows:

$$
f_{x}(x)=\sum_{i=1}^{n} a_{i} f_{i}(x)
$$

In the formula, $a_{i}$ is the proportion of the $i$ th distribution and meets $\sum_{i=1}^{n} a_{i}=1, f_{i}(x)$ is the probability density function of the $i$ th distribution. All $f_{i}(x)$ take normal distribution; the parameters in formula (1) are calculated by using nonlinear least squares method. The bimodal and tri-modal normal distribution are used to mid-span moment effect of $6 \mathrm{~m}$ and $16 \mathrm{~m}$ standard span bridges, respectively, the parameters are shown in Table 1 and Table 2, respectively, and the histogram and probability density curves are shown in Fig.1.

In order to see the fitting effect, the sample frequency curve, the bimodal and the tri-modal normal distribution fitting curve are plotted on the normal probability paper, as shown in Figure.2. As can be seen from the Figure.2, the middle part, bimodal and three peak distribution of the probability distribution curve can better be fitted, fitting effect of tri-modal normal distribution is slightly better than bimodal normal distribution, the fitting curve and sample point deviate greatly at the end of the sample, it shows that multi-peak normal distribution cannot accurately fit the tail of vehicle load effect sample. The parameters of probability distribution function of vehicle load are determined by the measured vehicle load, which is close to the peak value of density, and many measured data, the contribution to the parameters is large, the fitting accuracy is high, at both ends of the probability density curve, the data quantity is little, the contribution to the parameters is small. For the safety assessment of the bridge, it is most concerned about the few maximum sample values that deviate from the normal value, namely the tail of the sample. It can be seen that bimodal or tri-modal normal distribution cannot accurately simulate the load condition of overweight vehicles which deviate from normal values. 


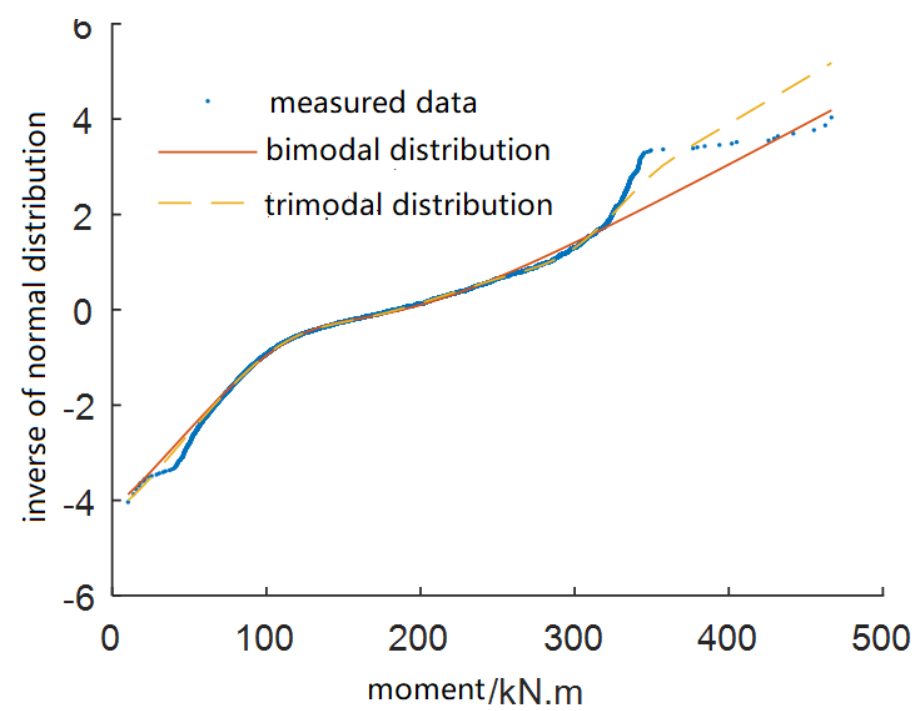

a) $6 \mathrm{~m}$ span

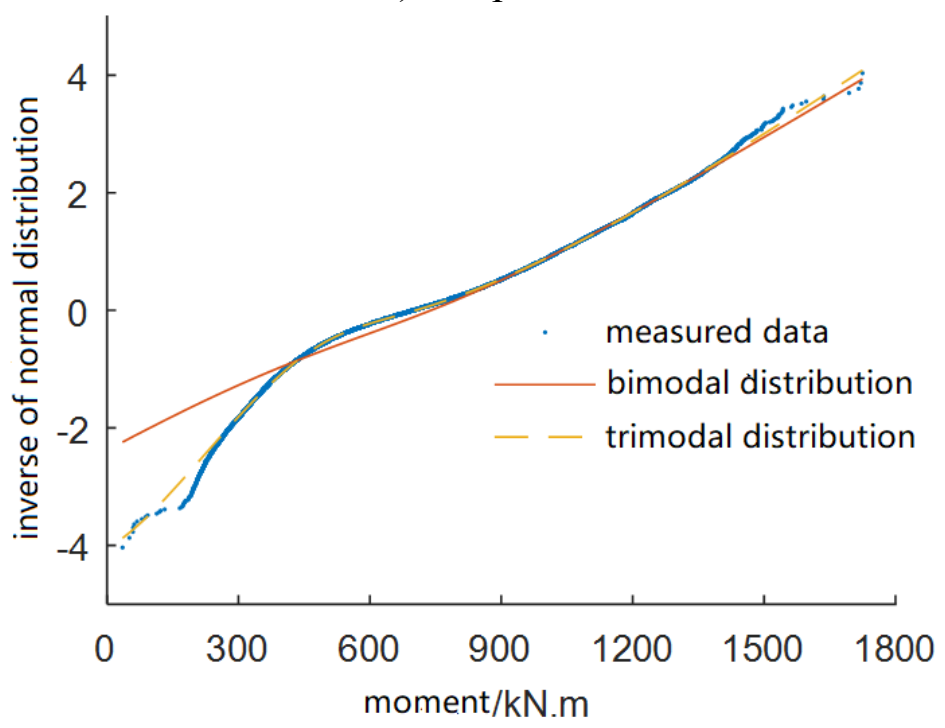

b) $16 \mathrm{~m}$ span

Fig.2 Cumulative probability curve for truck weight in normal probability paper Table.1 Parameter estimations of bimodal normal distribution

\begin{tabular}{cccc}
\hline $\mathrm{span} / \mathrm{m}$ & $\mathrm{i}$ & 1 & 2 \\
\hline \multirow{2}{*}{6} & $\mathrm{a}$ & 0.628 & 0.372 \\
& $\mu / \mathrm{kN} . \mathrm{m}$ & 235.07 & 103.89 \\
& $\sigma / \mathrm{kN} . \mathrm{m}$ & 56.74 & 24.81 \\
\multirow{2}{*}{16} & $\mathrm{a}$ & 0.374 & 0.626 \\
& $\mu / \mathrm{kN} . \mathrm{m}$ & 437.86 & 885.87 \\
& $\sigma / \mathrm{kN} . \mathrm{m}$ & 102.17 & 219.42 \\
\hline
\end{tabular}

Tab.2 Parameter estimations of trimodal normal distribution

\begin{tabular}{|ccccc|}
\hline span/m & $\mathrm{i}$ & 1 & 2 & 3 \\
& $\mathrm{a}$ & 0.146 & 0.509 & 0.345 \\
6 & $\mu / \mathrm{kN} . \mathrm{m}$ & 301.15 & 210.87 & 101.23 \\
& $\sigma / \mathrm{kN} . \mathrm{m}$ & 19.17 & 50.60 & 22.95 \\
& $\mathrm{a}$ & 0.372 & 0.275 & 0.353 \\
& $\mu / \mathrm{kN} . \mathrm{m}$ & 780.28 & 1001.88 & 432.48 \\
& $\sigma / k N . m$ & 202.16 & 191.03 & 98.78 \\
\end{tabular}




\section{The Tail Fitting of the Vehicle Load Effect Distribution}

In order to study the effect of tail fitting and trend, the piecewise function is used to fit distribution function of the sample. The middle part uses tri-modal normal distribution to fit, and the tail uses straight line and a parabola on the normal probability paper. The load effect distribution function of $6 \mathrm{~m}$ span bridge vehicles uses straight line to fit, the result is as follows:

$$
F(x)=\Phi(0.472 t+1.638)
$$

The parabola fitting is used; the tail function is as follows:

$$
F(x)=\Phi\left(0.303 t^{2}-1.947 t+6.405\right)
$$

In the formula 2 and 3, $\Phi()$ standard normal distribution function, $t=x / 100, x$ is the vehicle load effect, $x>350 \mathrm{kn} . \mathrm{M}$.

The $16 \mathrm{~m}$ span bridge uses the linear to fit, and the distribution function is as follows:

$$
F(x)=\Phi(0.292 t+1.151)
$$

The parabola fitting is used, and the distribution function is as follows:

$$
F(x)=\Phi\left(0.303 t^{2}-1.947 t+6.405\right)
$$

The symbol meaning is the same in formula 4 and $5, x>1500 \mathrm{kN} . \mathrm{m}$

\section{POT Model}

The POT model only studies the tail data of the sample, which does not need to assume its overall probability distribution, and it does not affected by its overall distribution. This paper also adopts POT model to fit the distribution curve of vehicle load effect sample. The basic concept of POT model is as follows:

It is assumed that $x_{1}, x_{2} \ldots \ldots x_{n}$ is the random variable of independent and identical distribution, and they are all subject to $F(x)$ distribution, $u$ indicate a sufficiently large threshold, Suppose the number of samples which exceed $u$ are $N$. If $x_{i}>u, x_{i}$ is called an ultra-threshold, $y_{i}=x_{i}-u$ called an excess. When $u$ is large enough, and the excess $y_{i}$ is subject to the generalized Pareto distribution (GPD ") [11]:

$$
\left\{\begin{array}{l}
F_{\varepsilon, \beta}(y)=1-\left(1+\frac{\xi}{\beta} y\right)^{-1 / \xi}, \xi \neq 0 \\
F_{\varepsilon, \beta}(y)=1-\exp \left(-\frac{y}{\sigma}\right), \xi=0
\end{array}\right.
$$

$\beta$ is the scale parameter, $\xi$ is the shape parameter. When $\xi=0$, GPD is degraded into is exponential distribution, when $\xi>0$, GPD is the long tail (thick tail) distribution, when $\xi<0$, GPD is truncated distribution.

The excess distribution of the threshold value of the random variable $x_{i}$ is the conditional excess distribution function:

$$
\begin{aligned}
F_{u}(y) & =P(X-u \leq y \mid X>u) \\
& =\frac{F(u+y)-F(u)}{1-F(u)}
\end{aligned}
$$

Through the transformation of the excess distribution function, the tail distribution of the vehicle load effect that exceeds the threshold is obtained:

$$
F(x)=[1-F(u)] \times F_{u}(y)+F(u)
$$

Among them, $x>u, 0 \leq y \leq x_{f}-u$ 
The kurtosis method [12] is used to determine the threshold value in this paper, and the maximum likelihood is used to calculate the GPD distribution parameters. Probability distribution function of vehicle load effect of $6 \mathrm{~m}$ span bridge:

$$
F(x)=1-0.00105[1+0.0052(x-342.36)]^{-5.6}
$$

In the formula (9), $x$ is the vehicle load effect, $x>342.36$.

$16 \mathrm{~m}$ span distribution function:

$$
F(x)=1-0.00069\left[1+0.0015(x-1512.72]^{-11.4}\right.
$$

In formula (10), $x$ is vehicle load effect, $x>1512.72 \mathrm{kN}$. M.

\section{Maximum Analysis in Reference Period}

Bridge assessment or reliability analysis need to design the probability distribution of the vehicle load maximum in reference period or assessment reference period, therefore, therefore, probability distribution function of vehicle load sample in this paper needs to be converted into the maximum distribution of the vehicle load design in reference period. According to mathematical statistics theory, if the probability distribution function of the random variable $X$ is $F_{X}(x)$, then the probability distribution function of maximum of independent and identically distributed random variable, $X_{1} X_{2} \quad \ldots \quad X_{m}$ are [13]

$$
F_{X, \max }(x)=\left[F_{X}(x)\right]^{m}
$$

WIM data can be used to accurately obtain the actual vehicle load of bridge during a certain period of time, according to the measured data of vehicle load during $n$ days, count vehicle load distribution, get the vehicle load distribution function, then the design maximum distribution function of reference period in $\mathrm{N}$ years [13]:

$$
F_{X, \max }(x)=\left[F_{1}(x)\right]^{365 N / n}
$$

$m=365 \mathrm{~N} / \mathrm{n}$, the he probability density function is:

$$
f_{N, \max }(x)=m f_{1}(x)\left[F_{X}(x)\right]^{m-1}
$$

The tri-modal distribution normal distribution is used to fit the middle part of the sample distribution curve, the bimodal normal distribution model, the tri-modal normal distribution model, linear fitting model, parabolic fitting model and tail POT model is used to fit tail of sample distribution curve, respectively, and calculate maximum vehicle load effect in 100 years' design reference period. The maximum distribution of vehicle load effect of $6 \mathrm{~m}$ span bridge as shown in Figure. $3 \mathrm{a}$, the maximum distribution of vehicle load effect of $6 \mathrm{~m}$ span bridge as shown in Figure. $3 \mathrm{~b}$, as can be seen from the Figure.3, using different methods to fit the distribution curve tail of vehicle load effect sample, there are significant differences in the distribution of maximum of vehicle load effect in reference period.

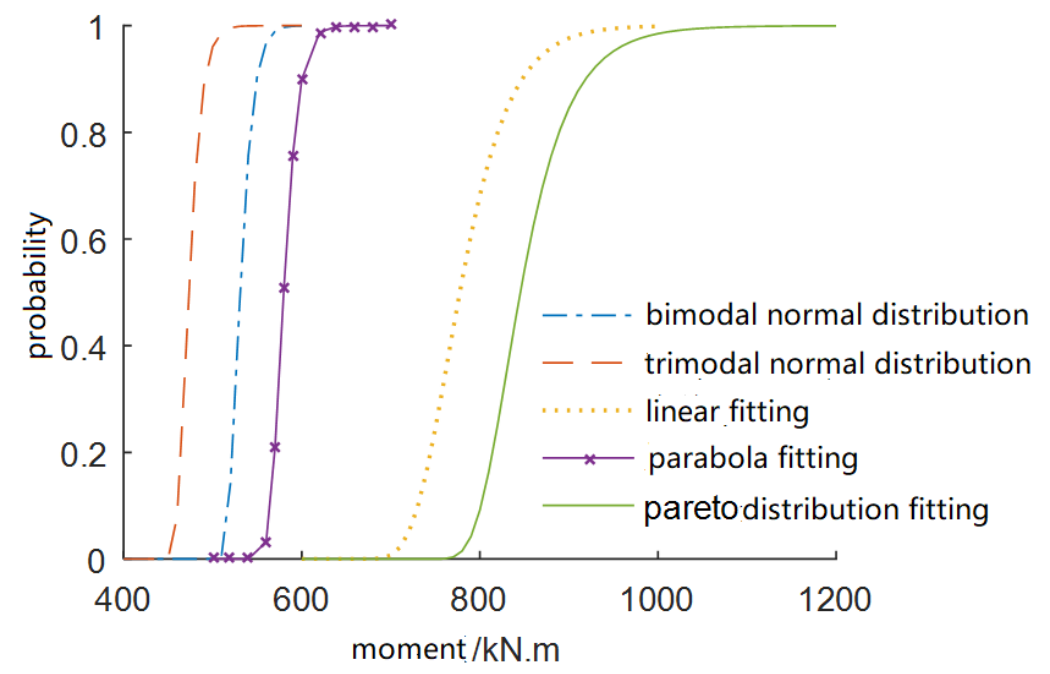

A) $6 \mathrm{~m}$ span 


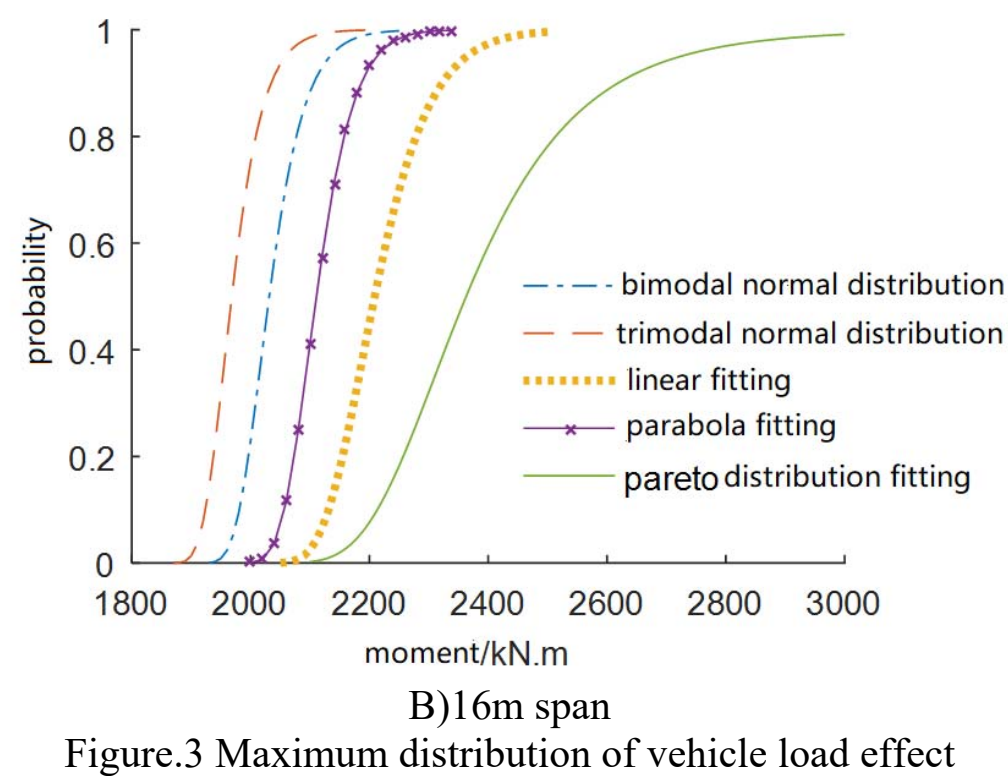

The corresponding load effect at 0.95 fractile is taken as the standard value, and the standard value of vehicle load effect in different reference periods are shown in Figure. 4. As can be seen from Figure.4.

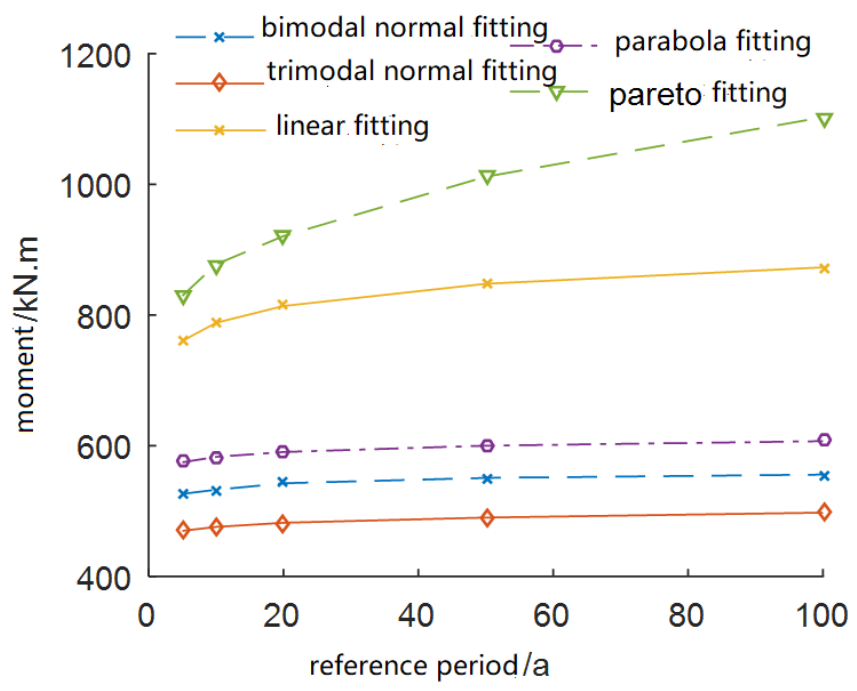

a) $6 \mathrm{~m}$ span

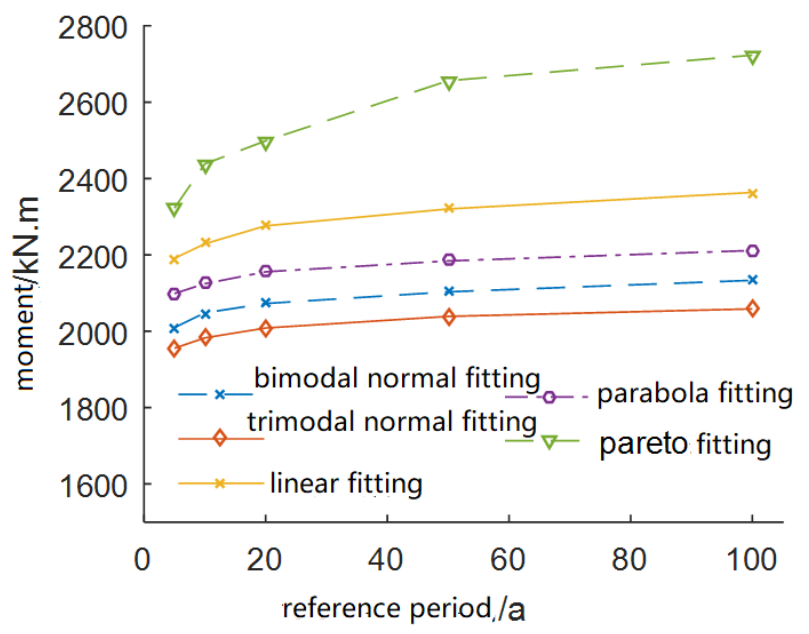

b) $16 \mathrm{~m}$ span

Figure.4 Characteristic value of moment

In order to study the effect of distribution curve tail fitting of vehicle load effect sample on the design reference period maximum, the vehicle probability distribution function of the measured 
samples, bimodal normal distribution model, tri-modal normal distribution model, linear fitting model, parabola fitting model and POT model is drawn on the normal probability paper, and observe its tail distribution. The $6 \mathrm{~m}$ span is shown in Figure 3.a, the $16 \mathrm{~m}$ span is shown in Figure 3.b.

As can be seen from Figure 3.a and Figure.3. b, the bimodal and tri-modal normal distribution model are seriously deviated from the tail of sample, and the convergence is the fastest. Using parabola fitting, the tail fitting of the measured data is better, but the trend over measured is not consistent with the measured data, and the convergence speed is accelerated with the increase of bending moment. The linear fitting, Pareto model are in consistent with the measured data tail, and the trend over the measured data is basically consistent with the measured data trend, the linear fitting model converges slightly faster than the POT model. Thus it can be seen

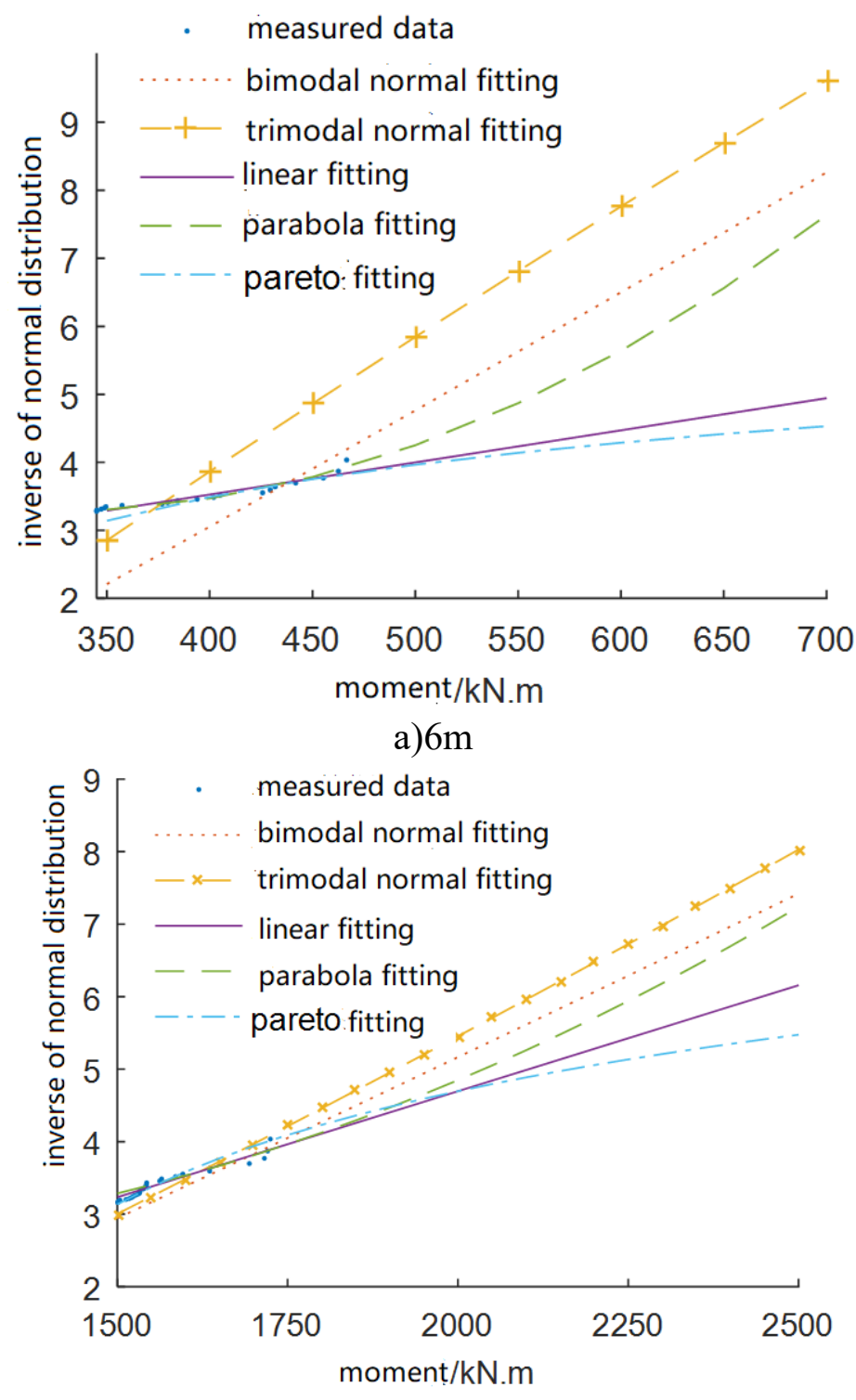

Figure.5 Tail fitting of truck effect sample

\section{Conclusion}

(1) The multimodal normal distribution model cannot accurately simulate the tail of the frequency curve of the vehicle load effect sample, and its use in extrapolation of the extreme of the vehicle load effect will cause a large error;

(2) The distribution key of the maximum of extrapolation vehicle load effect key in the reference period is to accurately fit tail of sample probability distribution curve, and determine the trend of the tail of the sample probability distribution curve, the tail trend of the probability distribution curve of the samples plays a decisive role in the extrapolation result of the extreme of the vehicle load effect. 


\section{Acknowledgements}

1. Guangxi Science and Technology Development project, research on the design theory and method of the highway concrete bridge widening and reconstruction, Guilin Science Breakthrough 1355008-9.

2. The National Natural Science Fund of China: research on vehicle load model on highway bridges affected by truck lateral distribution, 51468013 .

3. Guangxi Science and Technology Development Project, research and application of risk assessment methods for dangerous bridges and collapse risk, Guilin Science Breakthrough 14251012, main research.

\section{References}

[1]. The tail trend of the probability distribution curve of the samples plays a decisive role in the extrapolation result of the extreme of the vehicle load effect.

[2]. Ruan Xin, Zhou Junyong, Shi Xuefei. Review of the Extreme Value Extrapolation Method of Bridge Vehicle Load Response[J]. Journal of Tongji University (Natural Science Section), 2015, 43 (9): 1339-1346.

[3]. Li Yanghai, Bao Weigang, Guo Jianwu, etc. Reliability And Probability Limiting Condition Design of Highway Bridge[M]. Beijing: China Communications Press, 1997.

[4]. Mei Gang, Qin Quan, Lin Daojin. Bimodal Distribution Probability Model Of Vehicle Load On Highway Bridge [J]. Journal of Tsinghua university (Science and Technology), 2003, 43(10):1394-1397.

[5]. Gong Jinxin, Li Wenjie, Zhao Junli, Feng Min. Research on Vehicle Load Probability Model of Highway Bridge (1)- Non-governing over-speed areas. [J]. Journal of Highway and Transportation Research and Development, 2010, 27(6): 40-46.

[6]. Gong, Jinxin, Li Wenjie, Zhao Junli, Feng Min. Research on Vehicle Load Probability Model of Highway Bridge (2)-Toll-by-weight Area and Controlling Speeding Area [J]. Journal of Highway and Transportation Research and Development, 2010, 27(6): 40-46.

[7]. Han Dajian. Application and Comparison of Extreme Value Analysis Method in Vehicle Load Assessment[J]. Journal of Architecture and Science and Engineering, 2011, 2 8(2):11-13.

[8]. Zhou Xiaoyi. Statistical Analysis of Traffic Loads and Traffic Load Effects On Bridges [D]. Paris: University Paris-Est, 2013.

[9]. Li Zhihuai, Li Chun, Sun Jiankang, Li Quanwang. Maximum Estimation of Vehicle Load Effect Based On GPD Model[J]. Engineering Mechanics, 2012, 29(supplement I):166-171.

[10]. Yuan Weizhang, Huang Haiyun, Zhang Junping, Yin Xing, et al. Ehicle Load Extreme Prediction And Load Effect Of POT Model [J]. Journal of Henan University of Science and Technology: Natural Science, 2016, 37 (4):36-41.

[11]. PICKANDS J. Statistical Inference Using Extreme Order Statistics[J]. Annals of Statistics, 1975, 3(1):119-131.

[12]. Bin Kuanjiang, Cheng Bo, Wang Leilei Statistical Test on the Problem of Thickness and Thickness of Profit Distribution[J]. Statistics and Decision, 2009, 7; 83-85.

[13]. Fu Gongkang, John W. Shiling Pei, CSU), Reynaldo M. Pablo Jr Calibration for State of Michigan Trunkline Bridges[R]. Research Report RC-1466, Detroit,Michigan.

[14]. Andrzej S. Nowak* and Przemyslaw Rakoczy**WIM-Based Live Load for Bridges, KSCE Journal of Civil Engineering (2013) 17(3):568-574. 